\title{
DESIGN OF CANTILEVER BASED BIOSENSOR FOR BONE CANCER DETECTION
}

\author{
S.P. Balwir ${ }^{1}$, Mayur Sahare ${ }^{2}$, Ankush Raghuwanshi ${ }^{3}$, Snehal Angarwar $^{4}$, Rucha Fulzele ${ }^{5}$ \\ ${ }^{I}$ Assistant Professor Electronics Engineering, DBACER, Wanadongri Nagpur, Maharashtra, India \\ ${ }^{2}$ Electronics Engineering, RTMNU, DBACER, Wanadongri Nagpur, Maharashtra, India \\ ${ }^{3}$ Electronics Engineering, RTMNU, DBACER, Wanadongri Nagpur, Maharashtra, India \\ ${ }^{4}$ Electronics Engineering, RTMNU, DBACER, Wanadongri Nagpur, Maharashtra, India \\ ${ }^{5}$ Electronics Engineering, RTMNU, DBACER, Wanadongri Nagpur, Maharashtra, India
}

\begin{abstract}
This paper present the design of cantilever-based biosensors which proves to bean interesting research tool that is fast and cheap for the detection of bone cancer. In cantilever based biosensor, adevice dimension changes the sensitivity of the device reduces which is of prime important in biosensor. Micro Electro Mechanical System is constructed to achieve a certain engineering function by the integration of mechanical elements, sensors, actuators and electronics on a common silicon substrate through micro-fabrication technology. The cantilever will help in the early detection of bone cancer. The deflections in the cantilever caused by the stress and strain in the cantilever are studied for the detection of the bone cancer.
\end{abstract}

Keywords: Cantilever, Biosensor, Sensitivity, MEMS (Micro Electro Mechanical System), Stress, Strain, Bone Cancer.

\section{INTRODUCTION}

Micro Electro Mechanical System (MEMS) is the applcation of microsensors, transducers and actuators that can sense system environment and react to the changes in that environment with the use of a microcircuit control[1]. Micro Electro Mechanical System is the integration of active and passive elements on a single silicon substrate developed through more advanced IC processing technology. The BioMEMS based cantilever design is an advancement of the biosensor technology in MEMS. The analysis of biomolecular changes occurring during critical disease discovery and many problems in molecular biology can be studied through bioMEMS devices.

\subsection{Bone Cancer}

Bone cancer is formed in the cells of bones which is a rare type of cancer. An Osteosarcoma is the most common type of cancer that develops in bone. Like the osteoblasts in normal bone, the cells that form this cancer make bone matrix. But the bone matrix of the osteosarcoma is not as strong as that of normal bones. It is the most common histological form of primary bone cancer.

\subsection{Detection of Bone Cancer}

Vimentin is a protein that indicated the presence of Osteosarcoma. The transport of protein between the nucleus and plasma membrane is believed to be performed by the vimentin. It is expressed only during the early stages of cellular development and its replacement by tissue in later stages[2].
The molecular reaction takes place on the surface of cantilever which gives rise to immobilization. The immobilization is facilitated through absorption of amine rich polymer polyethylenemine (PEI). The reaction of the PEI adsorbs silicon oxide while reacting amine groups are released. The antibodies are bonded to the surface using EDC reagent in conjunction with $\mathrm{N}$-hydroxysuccinimide. The figure 1 shows the biomolecularreaction[2]. The reaction takes place between Carboxylic group of the antibody and the amines present on the surface[2].
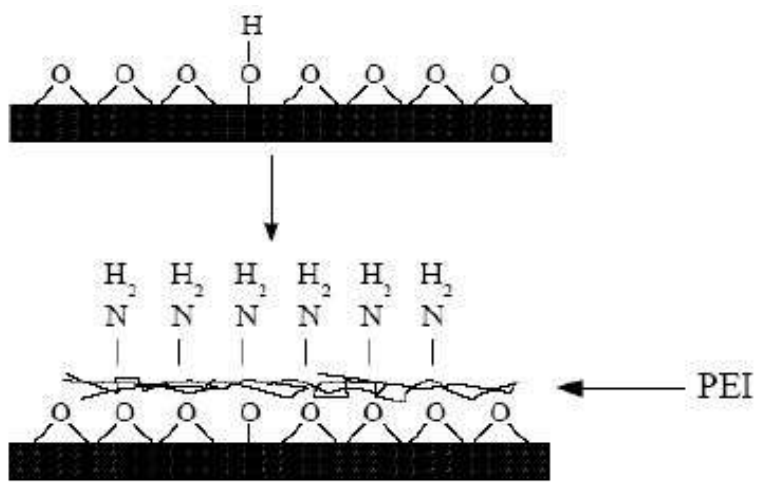

$\downarrow$
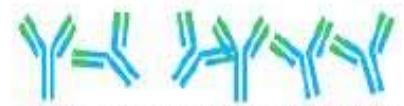

$\mathrm{NH} \mathrm{NH} \mathrm{NH} \mathrm{NH} \mathrm{NH} \mathrm{NH}$

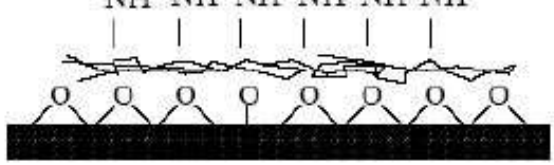

Fig(1):- Reaction on the surface of cantilever 


\subsection{Cantilever}

Cantilever responds mechanically to the change in stress on its surfaces and has a low flexural resistance. The bending of the cantilever due to the biochemical reactions on the surface which generate surface stress can be found by monitoring cantilever surface.

Due to the above mentioned chemical $\&$ biological reactions the cantilever gets deflected .This corresponding deflection in the cantilever is analyzed with change in its surface energy or tension. The concentration and attaching species can be determined by measuring the corresponding cantilever deflection

\section{MICRO-CANTILEVER-BASED BIOSENSOR}

The detection of an analyte, that combines a biological component with a physicochemical detector can be analytically detected by a biosensor. The new technology for detecting ultra small masses and biological specie has emerged in the form of Micro-cantilever sensors. Micro beams and cantilever structures are essentially very useful transducer elements using which many physical phenomena can be measured. The principle behind this lies with the deflection of beam and cantilever structures. The deflections are picked up either by capacitive or piezo resistive measurement. Firstly the molecular binding between the receptor and the biomolecules of interest and secondly, the read-out system capable of transducing the molecular binding in to detectable physical property are the two features that determine the success of the biological sensors.

\section{DESIGNING OF CANTILEVER}

The Cantilever design and simulation is carried out with Comsol4.4software. Comsol4.4 Multiphysics provides a platform for solving all kinds of scientific and engineering problems and is a powerful interactive environment for modeling.

The corresponding stress and voltage are studied in the cantilever design. For the study of cantilever we have designed various shapes of the cantilever design using COMSOL. The desired geometry of the cantilever should take less force and correspondingly give more displacement. The cantilever of Pi shape, E shape and rectangular shapes are studied and simulated using COMSOL. The comparative studies of the different designs of cantilever are done to judge which shape of the cantilever is best suited for the more precise computation.

The figure below shows the designs of cantilever using Comsol. The cantilever is made up of polysilicon material and sensitive layer of gold. The dimensions of the designed cantilever are $100 * 35 * 0.5 \mu \mathrm{m}$. The gap between the electrode and the beam is $1 \mu \mathrm{m}$.
The following design steps are employed in Comsol for designing the cantilever:-

1. To design the stationary solid structure by using the materials defined in the library of COMSOL.

2. Applying mesh to the structure for further analysis of result.

3. Design the cantilever structure by using the above two steps.

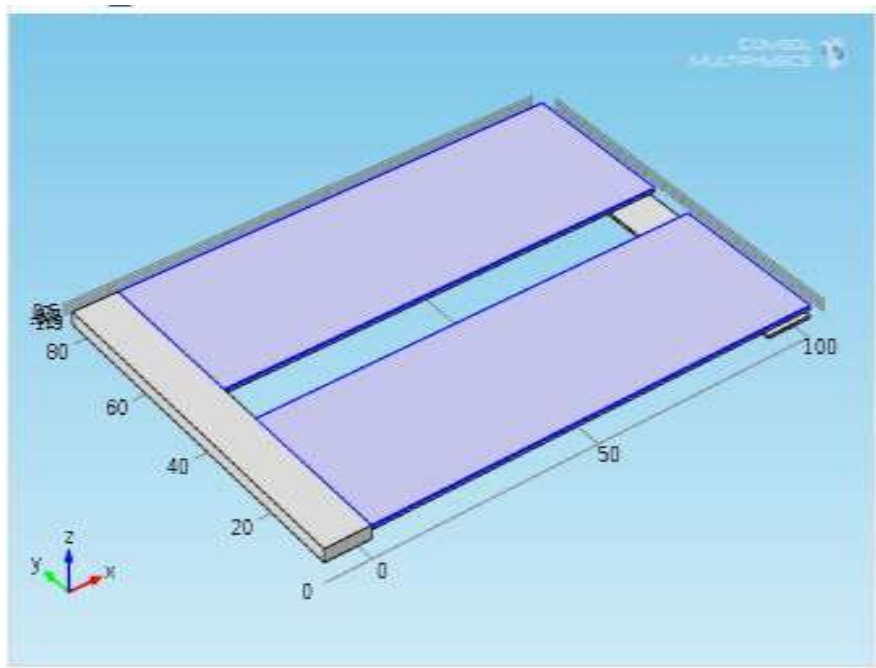

Fig (2):- Pi-shaped Cantilever

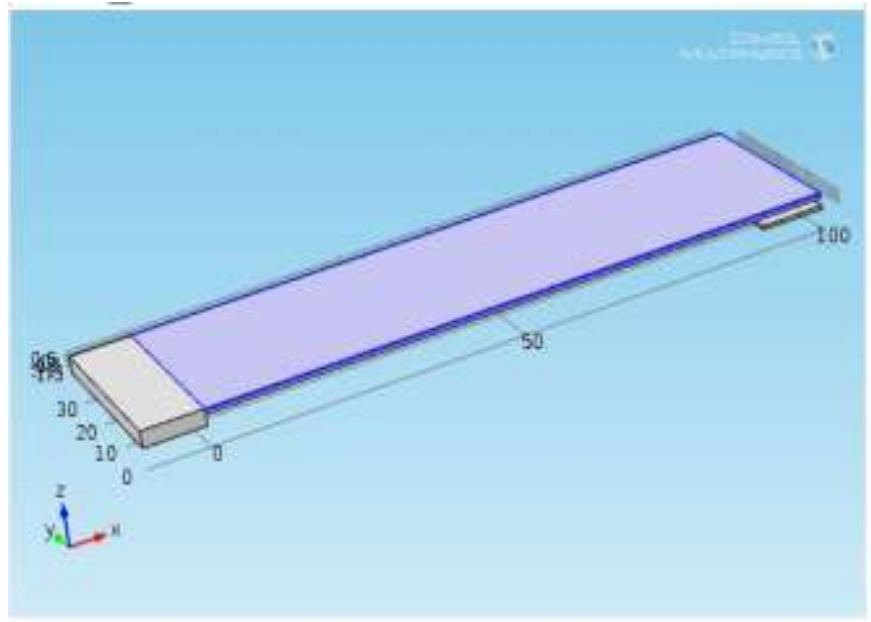

Fig (3):- Rectangular-shaped Cantilever

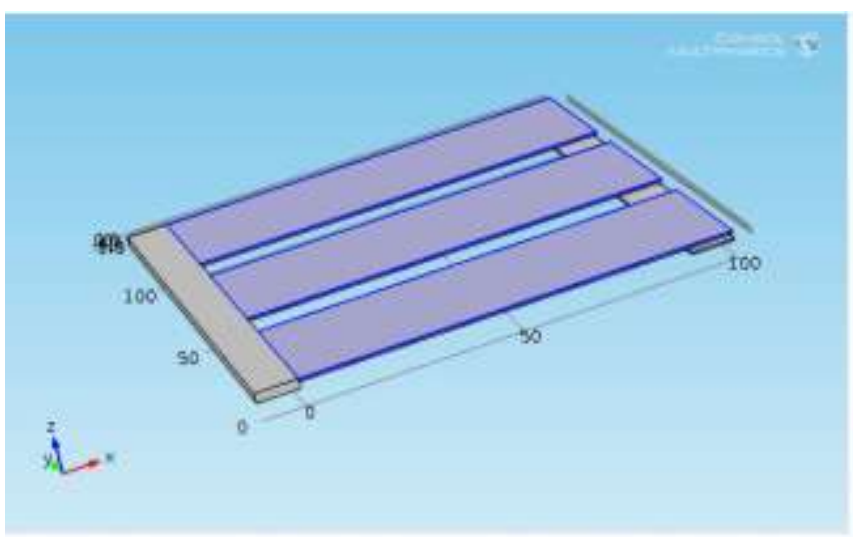

Fig (4):- E-shaped Cantilever 


\section{ANALYSIS OF THE CANTILEVER DESIGN}

The desired structure of cantilever is obtained using COMSOL4.4. In this design, beam will bend on applied electrostatic force by electrode. Beam is hanged by anchor where the anchor is the fixed part of the structure. It is to be noted that the electrode does not take part in the mechanical simulations that is it is not going to get deformed on the application of force, but it will participate in the electrostatic analysis. Mesh is an essential part of the simulation process, and can be crucially obtained for the best results in the fastest manner. In the given cantilever we apply a tetrahedral mesh to the active structure of the cantilever.

\begin{tabular}{|l|l|l|}
\hline $\begin{array}{l}\text { Sr. } \\
\text { no }\end{array}$ & Design Shape & $\begin{array}{l}\text { Stress } \\
(\text { in } \mu \mathrm{m})\end{array}$ \\
\hline 1 & Pi-shaped cantilever & $3.49 \times 10^{-9}$ \\
\hline 2 & $\begin{array}{l}\text { Rectangular } \\
\text { shaped cantilever }\end{array}$ & $3.5 \times 10^{-9}$ \\
\hline 3 & E shaped cantilever & $3.45 \times 10^{-9}$ \\
\hline
\end{tabular}

The table shows the value of stress developed on the surface of the cantilever. The applied force is $-1 * \mathrm{e}^{-6} \mathrm{~N} / \mathrm{m}^{2}$. The stress value is the most suited in the rectangular shaped cantilever. The rectangular shaped cantilever will be more suitable as its structure is simplified and less amount of sample is required for the detection.

The Stoney's formula issued to relate the cantilever end deflection and applied stress.

$$
\delta=\frac{3 \sigma(1-\mathrm{v})}{E}\left(\frac{\mathrm{L}}{\mathrm{t}}\right)^{2}
$$

In the above formula $\delta$ is the deflection of cantilever $\sigma$ is the stress on the cantilever $v$ is the Poission's ratio

Eis defined as Young's modulus

$\mathrm{L}$ gives the beam length

$\mathrm{t}$ gives the cantilever thickness

The physics employed in Solid Mechanics. The total force is being applied to the cantilever is correlated with the number of analyte molecules attraching to the gold surface of the cantilever[4].

The figure below shows the cantilever deflection results after simulation in COMSOL. The stress on the cantilever can be studied with respect to the deflections in the cantilever by these simulation results.

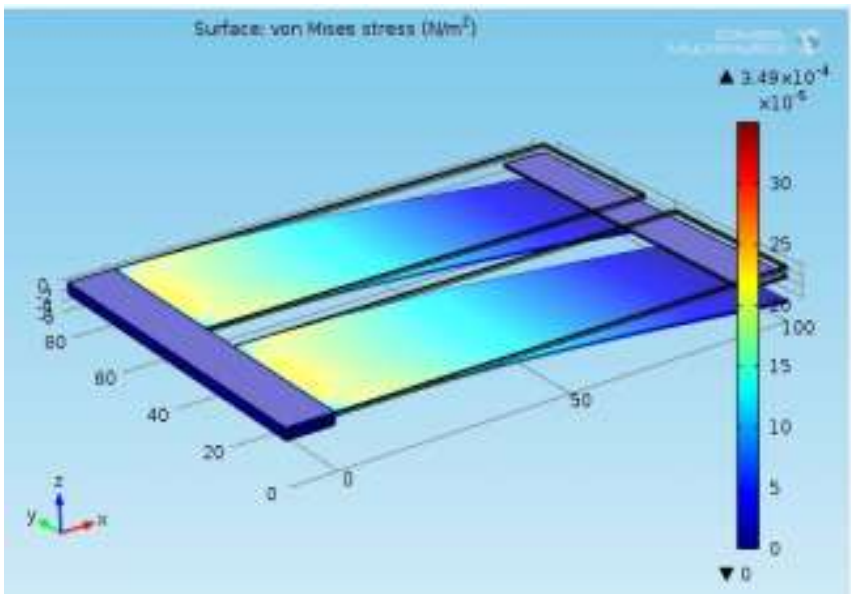

Fig (5):- Deflection in the Pi-shaped Cantilever

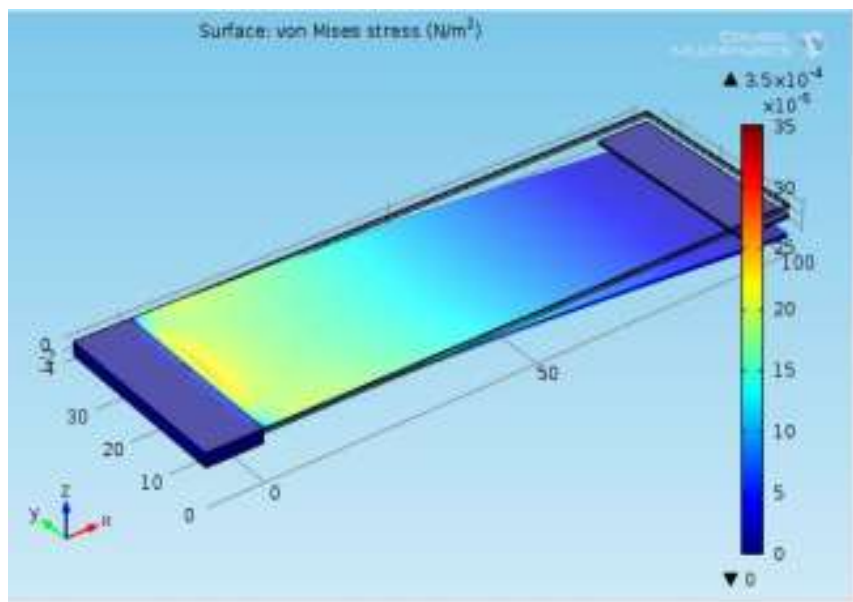

Fig (6):- Deflection in the Rectangular Cantilever

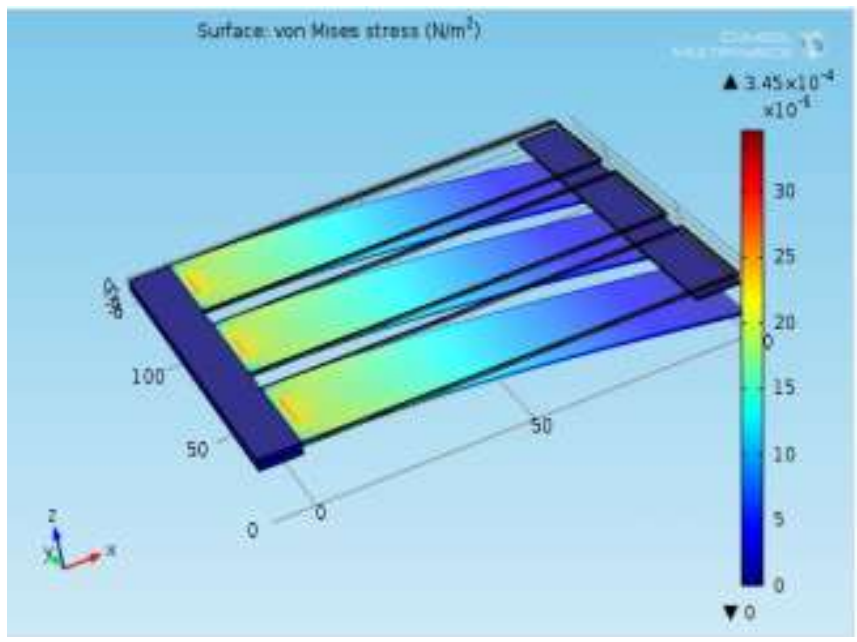

Fig (7):- Deflection in the E-shaped Cantilever

\section{RESULT AND CONCLUSION}

Various shapes of the cantilever are studied and the rectangular shaped cantilever is best suited of the evaluation. As the shape of the rectangular cantilever is more simplified than the Pi-shaped and E-shaped it is easy to construct. The amount of sample required for detection is less in case of rectangular shaped cantilever and it gives the précised results. The stress values of the cantilever are studied with 
respect to the deflection with the simulation results. The deflection results are studied with respect to the pull in voltage. The pull in voltage is the voltage at which the cantilever shows deflection. The applied voltage is being varied in between 5 Volts. The graph shows that it shows a deflection of $0.3 \mu \mathrm{m}$ at the pull in voltage. The pull in voltage is found to be 7.5 volts. After this voltage the cantilever shows the deflection. The graph below is ploted for the voltage verse displacement. This results are obtained after the simulation of the cantilever design in COMSOL.

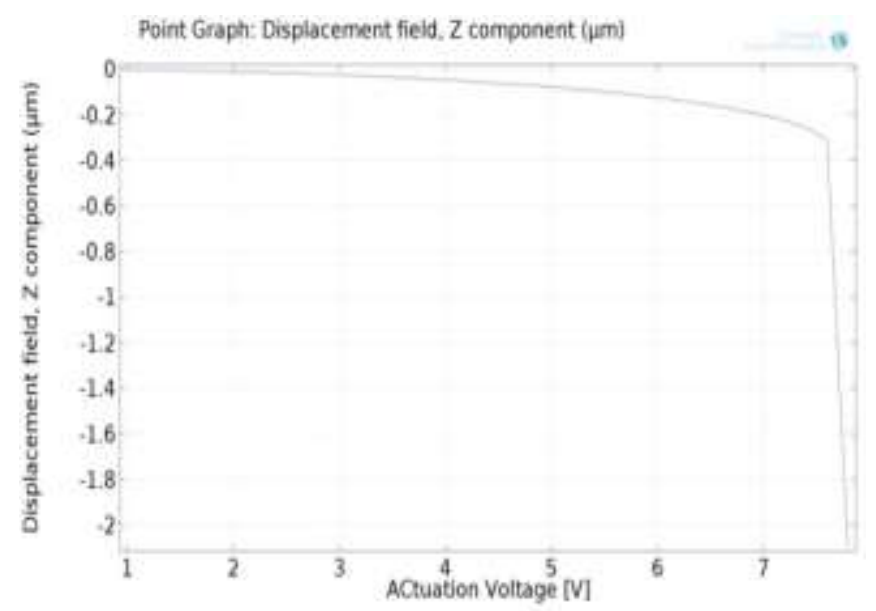

Fig(8):- Graph of voltage verses displacement

\section{REFERENCES}

[1] JayuKalambe, "Design and Simulation of Feedback Controller for Biosensor" International Conférence on VLSI, Communication \& Instrumentation (ICVCI) 2011 Proceedings published by International Journal of Computer Applications (IJCA).

[2] O. Bravo, C. Milburn, W.O.Soboyejo "Cantilever MEMS Detection of Cancer" Department of Mechanical and Aerospace Engineering Princeton University, Olden Street, Princeton, NJ 08544.

[3] Don Klaitabtim and AdisornTuantranont, "Design Consideration and Finite Element Modeling of MEMS Cantilever for Nano-Biosensor Applications", Proceedings of 2005 5th IEEE Conference on Nanotechnology Nagoya,Japan, July 2005.

[4] Saranya R, Saranya K, Ceemati D, MeenakshiSundaram N, "Design of MEMS Based Microcantilever for Tuberculosis Detection", Proceedings of 2013 COMSOL Conference in Banglore. 